\title{
EXTENSIONS OF SET-THEORETIC SOLUTIONS OF THE YANG-BAXTER EQUATION AND A CONJECTURE OF GATEVA-IVANOVA
}

\author{
L. VENDRAMIN
}

A Dino

\begin{abstract}
We develop a theory of extensions for involutive and nondegenerate solutions of the set-theoretic Yang-Baxter equation and use it to produce new families of solutions. As an application we construct an infinite family of counterexamples to a conjecture of Gateva-Ivanova related to the retractability of square-free solutions.
\end{abstract}

\section{INTRODUCTION}

The Yang-Baxter equation is one of the basic equations in mathematicalphysics. For that reason, in [9] Drinfeld posed the question of finding settheoretic solutions, i.e. bijective maps $r: X \times X \rightarrow X \times X$, where $X$ is a set, satisfying

$$
(r \times \text { id })(\text { id } \times r)(r \times \text { id })=(\text { id } \times r)(r \times \text { id })(\text { id } \times r) .
$$

Nondegenerate involutive solutions have received a lot of attention. Nondegenerate means that if one writes

$$
r(x, y)=\left(\sigma_{x}(y), \tau_{y}(x)\right), \quad x, y \in X,
$$

the maps $\sigma_{x}$ and $\tau_{x}$ are bijective for each $x \in X$. Involutive means that $r^{2}=\operatorname{id}_{X \times X}$.

The first results were obtained in the seminal works of Etingof, Schedler and Soloviev [10] and Gateva-Ivanova and Van den Bergh [17. The theory of involutive solutions was further developed in several papers such as [11,12], [18, 19], [13], [3], [14, 15], 4, 5], [8] and [7]. Most of these papers focus on the so-called square-free solutions, i.e. solutions satisfying $r(x, x)=(x, x)$ for all $x, y \in X$. Square-free solutions have several links with other topics (semigroups of I-type, semigroups of skew polynomial type, Bieberbach groups, quadratic algebras, etc.) and remarkable results were obtained. However, several important questions are still open. One of the main challenges is to construct new families of solutions. To understand how to construct new solutions, Etingof, Schedler and Soloviev considered an equivalence relation on $X$ which induces a natural involutive solution $\operatorname{Ret}(X, r)$, the so-called retraction of $(X, r)$. An involutive solution $(X, r)$ is then called a multipermutation solution of level $m$ if there exists a positive integer $m$ such that $\operatorname{Ret}^{m}(X, r)$ has only one element, where the iterated retractions are defined inductively as $\operatorname{Ret}^{k}(X, r)=\operatorname{Ret}\left(\operatorname{Ret}^{k-1}(X, r)\right)$ for $k>1$.

2010 Mathematics Subject Classification. 16T25.

This work was partially supported by CONICET, PICT-2014-1376 and ICTP. 
Multipermutation solutions has been intensively studied, see for example [4,5] and [13. In this context, one of the most important unsolved problems is the following:

Conjecture (Gateva-Ivanova). Every finite nondegenerate involutive squarefree set-theoretic solution $r: X \times X \rightarrow X \times X$ such that $|X| \geq 2$ is a multipermutation solution.

The conjecture was made in 2004, see [11, 2.28(I)].

The purpose of this note is to develop a theory of extensions of involutive nondegenerate set-theoretic solutions of the Yang-Baxter equation. To show the strength of the theory, we construct a set-theoretical solution that proves that [16. Conjecture 2.14] is not true and also answers [13, Open question 6.13(3)]. Another important application of our theory is the construction of counterexamples to the Gateva-Ivanova conjecture. One of these counterexamples is (up to isomorphism) the set-theoretic solution $(X, r)$, where $X=\{1,2, \ldots, 8\}$ and

$$
r(x, y)=\left(\varphi_{x}(y), \varphi_{y}(x)\right), \quad x, y \in X,
$$

with

$$
\begin{aligned}
& \varphi_{1}=(57), \quad \varphi_{2}=(68), \quad \varphi_{3}=(26)(48)(57), \quad \varphi_{4}=(15)(37)(68), \\
& \varphi_{5}=(13), \quad \varphi_{6}=(24), \quad \varphi_{7}=(13)(26)(48), \quad \varphi_{8}=(15)(24)(37) \text {. }
\end{aligned}
$$

Remarkably, extensions of this solution produce other counterexamples to the Gateva-Ivanova conjecture.

The paper is organized as follows. In Section 1 we review the basic notions of the set-theoretic Yang-Baxter equation. This section also includes the basics on cycle sets, which are nonassociative algebraic structures in bijective correspondence with nondegenerate involutive set-theoretic solutions. In Section 2 we develop the theory of extensions of cycle sets. Although these extensions are often hard to compute, they provide a very powerful tool to construct new solutions. Our main theorem appears in this section and states that if $X \rightarrow Y$ is a surjective homomorphism of cycle sets such that all the fibers have the same cardinality then $X$ is an extension of $Y$. In Section 3 several examples of extensions are given. Among these examples one finds the semidirect product of cycle sets and several classes of extensions that are relatively easy to compute. We conclude the paper with counterexamples to the Gateva-Ivanova conjecture.

\section{Preliminaries}

Recall that a pair $(X, r)$, where $X$ is a nonempty set and

$$
r: X \times X \rightarrow X \times X, \quad r(x, y)=\left(\sigma_{x}(y), \tau_{y}(x)\right), \quad x, y \in X,
$$

is a bijective map, is a set-theoretic solution of the Yang-Baxter equation if

$$
r_{12} r_{23} r_{12}=r_{23} r_{12} r_{23},
$$

where $r_{12}=r \times$ id and $r_{23}=\mathrm{id} \times r$. The map $r$ is involutive if $r^{2}=\mathrm{id}_{X \times X}$, it is nondegenerate if the maps $\sigma_{x}$ and $\tau_{x}$ are bijective for each $x \in X$, and it is square-free if $r(x, x)=(x, x)$ for all $x \in X$. 
A homomorphism between the set-theoretic solutions $\left(X, r_{X}\right)$ and $\left(Y, r_{Y}\right)$ is a map $f: X \rightarrow Y$ such that

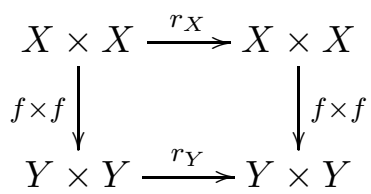

is commutative. An isomorphism of solutions is just a bijective homomorphism of solutions.

Convention 1.1. By a solution (of the Yang-Baxter equation) we mean a nondegenerate involutive set-theoretic solution of the Yang-Baxter equation. We consider only finite solutions.

The retract relation on a solution $(X, r)$ was first considered in [10] and it is defined by $x \sim y$ if and only if $\sigma_{x}=\sigma_{y}, x, y \in X$. The retraction of $(X, r)$ is the solution $\operatorname{Ret}(X, r)=(X / \sim, \bar{r})$ with

$$
\bar{r}([x],[y])=\left(\left[\sigma_{x}(y)\right],\left[\tau_{y}(x)\right]\right),
$$

where $[x]$ denotes the equivalence class of $x$. A solution $(X, r)$ is called a multipermutation solution of level $\operatorname{mpl} X=m$ if there exists $m>0$ such that $\operatorname{Ret}^{m}(X, r)$ has cardinality one, where

$$
\operatorname{Ret}^{k}(X, r)=\operatorname{Ret}\left(\operatorname{Ret}^{k-1}(X, r)\right), \quad k>0 .
$$

The Yang-Baxter permutation group $\mathcal{G}(X, r)$ of a finite solution $(X, r)$ is the subgroup of the symmetric group on $X$ generated by $\left\{\sigma_{x}: x \in X\right\}$.

Example 1.2. The map

$$
r(x, y)=\left(\sigma_{x}(y), \tau_{y}(x)\right), \quad x, y \in\{1,2,3,4\},
$$

where

$$
\begin{array}{llll}
\sigma_{1}=(34), & \sigma_{2}=(1324), & \sigma_{3}=(1423), & \sigma_{4}=(12), \\
\tau_{1}=(24), & \tau_{2}=(1432), & \tau_{3}=(1234), & \tau_{4}=(13),
\end{array}
$$

is a solution of the Yang-Baxter equation. It is not square-free and it is not a multipermutation solution. Moreover, $\mathcal{G}(X, r) \simeq \mathbb{D}_{4}$, where $\mathbb{D}_{4}$ is the dihedral group of eight elements.

To study solutions of the Yang-Baxter equation, Rump introduced what is known as cycle sets [18]. A cycle set is a set $X$ with a binary operation $X \times X \rightarrow X,(x, y) \mapsto x \cdot y$, such that the maps $\varphi_{x}: y \mapsto x \cdot y$ are bijective for each $x \in X$ and

$$
(x \cdot y) \cdot(x \cdot z)=(y \cdot x) \cdot(y \cdot z)
$$

for all $x, y, z \in X$. To describe a finite cycle set $X$, without loss of generality we may assume that $X=\{1,2, \ldots, n\}$ for some $n \in \mathbb{N}$ and then write the sequence of permutations $\varphi_{1}, \varphi_{2}, \ldots, \varphi_{n}$.

Cycle sets are in bijective correspondence with involutive nondegenerate solutions of the Yang-Baxter equation. The correspondence is given by

$$
r(x, y)=((y * x) \cdot y, y * x), \quad x, y \in X,
$$


where $y * x=z$ if and only if $x=y \cdot z$, see [18, Prop. 1]. From the correspondence (1.1) one obtains immediately that $r$ is square-free if and only if $x \cdot x=x$ for all $x \in X$. Thus we say that a cycle set $X$ is square-free if $x \cdot x=x$ for all $x \in X$.

A homomorphism between the cycle sets $X$ and $Z$ is a map $f: X \rightarrow Z$ such that $f(x \cdot y)=f(x) \cdot f(y)$ for all $x, y \in X$. As usual, an isomorphism of cycle sets is just a bijective homomorphism of cycle sets.

Example 1.3. The square-free solution over $X=\{1,2,3\}$ given by the map $r: X \times X \rightarrow X \times X$, where

$$
\begin{array}{lll}
r(1,1)=(1,1), & r(1,2)=(2,1), & r(1,3)=(3,2), \\
r(2,1)=(1,2), & r(2,2)=(2,2), & r(2,3)=(3,1), \\
r(3,1)=(2,3), & r(3,2)=(1,3), & r(3,3)=(3,3),
\end{array}
$$

has permutations

$$
\sigma_{1}=\sigma_{2}=\tau_{1}=\tau_{2}=\mathrm{id}, \quad \sigma_{3}=\tau_{3}=(12) .
$$

This solution corresponds to the square-free cycle set $X=\{1,2,3\}$ given by the permutations $\varphi_{1}=\varphi_{2}=\mathrm{id}$ and $\varphi_{3}=(12)$.

Notation 1.4. For a nonempty set $S$ we write $\operatorname{Fun}(S, S)$ to denote set of maps $S \rightarrow S$ and $\operatorname{Sym}(S)$ to denote the set of permutations on $S$. We usually write $f(-)$ to denote the map $t \mapsto f(t)$. We write $|X|$ to denote the cardinality of the set $X$.

\section{Dynamical extensions}

Inspired by the theory of extensions for racks and quandles [1, §2], we develop a theory of dynamical extensions of finite cycle sets.

Lemma 2.1. Let $X$ be a finite cycle set, $S$ be a finite nonempty set, and $\alpha: X \times X \times S \rightarrow \operatorname{Fun}(S, S),(x, y, s) \mapsto \alpha_{x, y}(s,-)$, be a map. Then $S \times X$ is a cycle set with respect to

$$
(s, x) \cdot(t, y)=\left(\alpha_{x, y}(s, t), x \cdot y\right), \quad x, y \in X, s, t \in S,
$$

if and only if the maps $t \mapsto \alpha_{x, y}(s, t)$ are bijective for each $x, y \in X$ and $s \in S$, and

$$
\alpha_{x \cdot y, x \cdot z}\left(\alpha_{x, y}(r, s), \alpha_{x, z}(r, t)\right)=\alpha_{y \cdot x, y \cdot z}\left(\alpha_{y, x}(s, r), \alpha_{y, z}(s, t)\right)
$$

holds for all $x, y, z \in X$ and $r, s, t \in S$.

Proof. It is straightforward to check that the operation (2.1) is invertible if and only if the maps $t \mapsto \alpha_{x, y}(s, t)$ are invertible for each $x, y \in X$ and $s \in S$. Then

$$
(r, x) \cdot(s, y)=(t, z) \Longleftrightarrow(s, y)=\left(\alpha_{x, x * z}^{-1}(r, t), x * z\right),
$$

where $*$ denotes the inverse of the binary operation of the cycle set $X$.

Now for $(r, x),(s, y),(t, z) \in S \times X$ one computes

$$
\begin{aligned}
((r, x) \cdot(s, y)) & \cdot((r, x) \cdot(t, z)) \\
& =\left(\alpha_{x, y}(r, s), x \cdot y\right) \cdot\left(\alpha_{x, z}(r, t), x \cdot z\right) \\
& =\left(\alpha_{x \cdot y, x \cdot z}\left(\alpha_{x, y}(r, s), \alpha_{x, z}(r, t)\right),(x \cdot y) \cdot(x \cdot z)\right)
\end{aligned}
$$


and similarly

$$
((s, y) \cdot(r, x)) \cdot((s, y) \cdot(t, z))=\left(\alpha_{y \cdot x, y \cdot z}\left(\alpha_{y, x}(s, r), \alpha_{y, z}(s, t)\right),(y \cdot x) \cdot(y \cdot z)\right) .
$$

Then the lemma follows.

Definition 2.2. Let $X$ be a finite cycle set and $S$ a nonempty finite set. A map $\alpha: X \times X \times S \rightarrow \operatorname{Sym}(S)$ satisfying (2.2) is called a dynamical cocycle of $X$ with values on $S$.

Notation 2.3. We write $Z^{2}(X, S)$ to denote the set of dynamical cocycles of the finite cycle set $X$ with values in the finite set $S$, i.e.

$$
Z^{2}(X, S)=\{\alpha: X \times X \times S \rightarrow \operatorname{Fun}(S, S): \alpha \text { is a dynamical cocycle }\} .
$$

Definition 2.4. Let $X$ be a finite cycle set, $S$ a nonempty finite set and $\alpha \in Z^{2}(X, S)$. The cycle set $S \times_{\alpha} X$ constructed by Lemma 2.1 is called a dynamical extension of $X$ by $\alpha$.

Example 2.5. Let $X$ be a finite cycle set $X$ and $S$ a finite nonempty set. The map $\alpha: X \times X \rightarrow \operatorname{Sym}(S)$ given by $\alpha_{x, y}(s, t)=t$ for all $x, y \in X$ and $s, t \in S$ is a dynamical cocycle of $X$. This is the trivial dynamical cocycle of $X$.

Remark 2.6. If $X$ is a finite square-free cycle set, $S$ is a nonempty finite set and $\alpha \in Z^{2}(X, S)$, then $S \times_{\alpha} X$ is square-free if and only if

$$
\alpha_{x, x}(s, s)=s \quad \text { for all } x \in X \text { and } s \in S .
$$

Definition 2.7. We say that a homomorphism $p: X \rightarrow Y$ between the finite cycle sets $X$ and $Y$ is a covering map if it is surjective and all the fibers $p^{-1}(y)$, where $y \in Y$, have the same cardinality.

Definition 2.8. A covering map $X \rightarrow Y$ is trivial if either $|Y|=1$ or $|Y|=|X|$.

Definition 2.9. A finite cycle set $X$ is simple if $|X|>1$ and any covering map $X \rightarrow Y$ is trivial.

Example 2.10. Every cycle set with a prime number of elements is simple.

Example 2.11. Let $X=\{1,2,3,4\}$ be the cycle set given by

$$
\varphi_{1}=(14), \quad \varphi_{2}=(1342), \quad \varphi_{3}=(23), \quad \varphi_{4}=(1243) .
$$

Let us prove that $X$ is simple. If $p: X \rightarrow Y$ is a covering map, then $|Y|=2$. Thus $Y=\{a, b\}$ with $a \neq b$. Since there are only two cycle sets with two elements, there are two cases to consider.

Suppose first that the cycle set structure over $Y$ is given by $\psi_{a}=\psi_{b}=\mathrm{id}$. Since $p$ is a cycle set homomorphism and $\psi_{a}=\psi_{b}=\mathrm{id}$, it follows that $p(x \cdot x)=p(x) \cdot p(x)=p(x)$ for all $x \in X$. But $x \cdot x \neq x$ for all $x \in X$ and hence $p(x)=p(y)$ for all $x, y \in X$, a contradiction.

Now suppose that $\psi_{a}=\psi_{b}=(a b)$ and $p(1)=a$. From $1 \cdot 1=4$ one obtains that $p(4)=b$ and hence $p(2)=p(4 \cdot 1)=p(4) \cdot p(1)=b \cdot a=b$. Then $a=a \cdot b=p(1) \cdot p(2)=p(1 \cdot 2)=p(2)=b$, a contradiction. 
Theorem 2.12. Let $X$ be a finite cycle set and assume that $X$ admits a covering map $p: X \rightarrow Y$ onto a finite cycle set $Y$. Then there exists a finite nonempty set $S$ and a dynamical cocycle $\alpha \in Z^{2}(X, S)$ such that $X$ is isomorphic to the dynamical extension $S \times_{\alpha} Y$.

Proof. Since all the fibers $p^{-1}(y)$ have the same cardinality, there is a finite nonempty set $S$ and there are bijections $f_{y}: p^{-1}(y) \rightarrow S$ for each $y \in Y$. Let $\alpha: Y \times Y \times S \rightarrow \operatorname{Fun}(S, S)$ be the map given by

$$
\alpha_{y, z}(s, t)=f_{y \cdot z}\left(f_{y}^{-1}(s) \cdot f_{z}^{-1}(t)\right), \quad s, t \in S, y, z \in Y .
$$

Then $\alpha \in Z^{2}(Y, S)$ and hence $S \times Y$ is a cycle set with respect to

$$
(s, y) \cdot(t, z)=\left(\alpha_{y, z}(s, t), y \cdot z\right), \quad s, t \in S, y, z \in Y .
$$

The map $\phi: X \rightarrow S \times Y$ given by $x \mapsto\left(f_{p(x)}(x), p(x)\right)$ is a homomorphism of cycle sets. Furthermore, $\phi$ is bijective since the map $\psi: S \times Y \rightarrow X$ given by $\psi(s, y)=f_{y}^{-1}(s)$ satisfy $\phi \circ \psi=\operatorname{id}_{S \times Y}$ and $\psi \circ \phi=\operatorname{id}_{X}$.

Corollary 2.13. Let $X$ be a finite nonsimple cycle set. Then there exists a finite cycle set $Y$ with $1<|Y|<|X|$, a finite nonempty set $S$ and a dynamical cocycle $\alpha \in Z^{2}(X, S)$ such that $X$ is isomorphic to $S \times_{\alpha} Y$.

Proof. If follows immediately from Theorem 2.12 .

Example 2.14. Let $X=\{1, \ldots, 6\}$ be the cycle set given by

$$
\varphi_{1}=\varphi_{2}=(12)(34)(56), \quad \varphi_{3}=\varphi_{5}=(12)(3654), \quad \varphi_{4}=\varphi_{6}=(12)(3456),
$$

and let $Y=\{1,2\}$ be the cycle set given by

$$
\psi_{1}=\psi_{2}=(12) \text {. }
$$

The map

$$
p: X \rightarrow Y, \quad p(x)= \begin{cases}1 & \text { if } x \text { is odd }, \\ 2 & \text { otherwise },\end{cases}
$$

is a surjective cycle set homomorphism. Since the fibers $p^{-1}(1)$ and $p^{-1}(2)$ have both three elements, Theorem 2.12 implies that there exists $S=\{a, b, c\}$ and $\alpha \in Z^{2}(X, S)$ such that $X \simeq S \times{ }_{\alpha} Y$. The bijections

$$
f_{1}: 1 \mapsto a, 3 \mapsto b, 5 \mapsto c, \quad f_{2}: 2 \mapsto a, 4 \mapsto b, 6 \mapsto c,
$$

and a direct calculations yield

$$
\alpha_{x, y}(s,-)= \begin{cases}(b c) & \text { if }(x, y, s) \in\{(1,1, b),(1,1, c),(2,2, b),(2,2, c)\} \\ \text { id } & \text { otherwise. }\end{cases}
$$

Definition 2.15. Two dynamical cocycles $\alpha, \beta \in Z^{2}(X, S)$ are cohomologous if there exists a map $\gamma: X \rightarrow \operatorname{Sym}(S), x \mapsto \gamma_{x}$, such that

$$
\gamma_{x \cdot y}\left(\alpha_{x, y}\left(\gamma_{x}^{-1}(s), \gamma_{y}^{-1}(t)\right)\right)=\beta_{x, y}(s, t)
$$

for all $x, y \in X$ and $s, t \in S$.

Proposition 2.16. Let $X$ be a finite cycle set, $S$ a nonempty finite set and $\alpha, \beta \in Z^{2}(X, S)$. The following hold: 
(1) If $\alpha$ and $\beta$ are cohomologous by $\gamma$, then

$$
F: S \times_{\alpha} X \rightarrow S \times_{\beta} X, \quad(s, x) \mapsto\left(\gamma_{x}(s), x\right),
$$

is a bijective cycle set homomorphism.

(2) If there is a bijective cycle set homomorphism $F: S \times_{\alpha} X \rightarrow S \times{ }_{\beta} X$ such that $p_{\beta} \circ F=p_{\alpha}$, where $p_{\alpha}: S \times_{\alpha} X \rightarrow X$ and $p_{\beta}: S \times_{\beta} X \rightarrow X$ are the canonical surjections, then $\alpha$ and $\beta$ are cohomologous.

Proof. We first prove (1). Since $\gamma_{x} \in \operatorname{Sym}(S)$ for all $x \in X$, the map $F$ is bijective. Let us prove that $F$ is a cycle set homomorphism: For $(s, x),(t, y) \in S \times X$ one computes

$$
F((s, x) \cdot(t, y))=F\left(\alpha_{x, y}(s, t), x \cdot y\right)=\left(\gamma_{x \cdot y}\left(\alpha_{x, y}(s, t)\right), x \cdot y\right)
$$

and similarly

$$
F(s, x) \cdot F(t, y)=\left(\gamma_{x}(s), x\right) \cdot\left(\gamma_{y}(t), y\right)=\left(\beta_{x, y}\left(\gamma_{x}(s), \gamma_{y}(t)\right), x \cdot y\right) .
$$

Thus the claim follows from Equation (2.3).

Now we prove (2). Since $F$ is bijective and $p_{\beta} \circ F=p_{\alpha}$, we may assume that $F(s, x)=(f(s, x), x)$ for some $f: S \times X \rightarrow S$. Then the maps $\gamma_{x}: S \rightarrow S, s \mapsto f(s, x)$, are bijective for each $x \in X$. Since $F$ is a cycle set homomorphism, the claim follows from Equations (2.4) and (2.5).

\section{EXAMPLES}

In this section we collect some examples of (dynamical) cocycles and (dynamical) extensions of cycle sets.

Example 3.1 (Rump's semidirect product of cycle sets). Let $X$ and $S$ be finite cycle sets and suppose that $X$ acts on $S$, which means that there is a map $X \times S \rightarrow S,(x, s) \mapsto x s$, such that

(1) $x(s \cdot t)=x s \cdot x t$ for all $x \in X$ and $s, t \in S$,

(2) $(x \cdot y) x s=(y \cdot x) y s$ for all $x, y \in X$ and $s \in S$, and

(3) The map $S \rightarrow S, s \mapsto x s$, is bijective for all $x \in X$.

$B y$ [20, Thm. 1], $S \times X$ is a cycle set with

$$
(s, x) \cdot(t, y)=((x \cdot y) s \cdot(y \cdot x) t, x \cdot y), \quad(s, x),(t, y) \in S \times X .
$$

From Lemma 2.1 one obtains that the map $\alpha: X \times X \times S \rightarrow \operatorname{Fun}(S, S)$ given by $\alpha_{x, y}(s, t)=(x \cdot y) s \cdot(y \cdot x) t$ is then a dynamical cocycle over $X$.

Let $\mathbf{n}_{m}$ be defined as the minimal integer so that there exists a squarefree multipermutation solution $X$ of size $\mathbf{n}_{m}$ and $\mathrm{mpl} X=m$. In [13, Open question 6.13(3)] it was asked if $\mathbf{n}_{\mathbf{m}}=2^{m-1}+1$ holds for all $m \in \mathbb{N}$.

Example 3.2. Let $Y=\{1,2,3\}$ be the cycle set given by $\varphi_{1}=\varphi_{2}=\mathrm{id}$, $\varphi_{3}=(12)$ and $S=\{a, b\}$ be a set with two elements. Write

$$
\mathcal{A}=\{(1,2, a),(1,2, b),(1,3, b),(2,1, a),(2,1, b),(2,3, b)\} \subseteq X \times X \times S .
$$

The map $\alpha: Y \times Y \times S \rightarrow \operatorname{Sym}(S)$ given by

$$
\alpha_{x, y}(s,-)= \begin{cases}(a b) & \text { if }(x, y, s) \in \mathcal{A}, \\ \text { id } & \text { otherwise },\end{cases}
$$


is a dynamical cocycle of $Y$. Let

$$
x_{j}= \begin{cases}(a, j) & \text { if } j \in\{1,2,3\}, \\ (b, j-3) & \text { if } j \in\{4,5,6\} .\end{cases}
$$

The extension $X=S \times_{\alpha} Y$ is the cycle set $\left\{x_{1}, \ldots, x_{6}\right\}$ given by

$$
\begin{aligned}
\psi_{x_{1}}=\left(x_{2} x_{5}\right), \quad \psi_{x_{2}}=\left(x_{1} x_{4}\right), \quad \psi_{x_{3}}=\left(x_{1} x_{2}\right)\left(x_{4} x_{5}\right), \\
\psi_{x_{4}}=\left(x_{2} x_{5}\right)\left(x_{3} x_{6}\right), \quad \psi_{x_{5}}=\left(x_{1} x_{4}\right)\left(x_{3} x_{6}\right), \quad \psi_{x_{6}}=\left(x_{1} x_{2}\right)\left(x_{4} x_{5}\right) .
\end{aligned}
$$

This cycle set corresponds to a square-free multipermutation solution of level four. This solution satisfies $4=\mathrm{mpl} X>\log _{2} 6$ and hence [16. Conjecture 2.14] is not true. This example also shows that $\mathbf{n}_{4} \leq 6<2^{3}+1$ and answers [13, Open question 6.13(3)].

3.1. Constant cocycles. A very interesting class of dynamical cocycles consists of the so-called constant cocycles. By definition, a constant cocycle $\alpha$ can be written as $\alpha_{x, y}(s, t)=\beta_{x, y}(t)$ for some $\beta: X \times X \rightarrow \operatorname{Sym}(S)$. For constant cocycles the cocycle condition (2.2) is

$$
\beta_{x \cdot y, x \cdot z} \beta_{x, z}=\beta_{y \cdot x, y \cdot z} \beta_{y, z}
$$

for all $x, y, z \in X$.

Example 3.3. Let $X=\{1,2,3\}$ be the square-free cycle set given by

$$
\varphi_{1}=\varphi_{2}=\mathrm{id}, \quad \varphi_{3}=(12),
$$

and let $S=\{a, b\}$ be a set with two elements. The map $\beta: X \times X \rightarrow \operatorname{Sym}(S)$ given by

$$
\beta_{1,1}=\beta_{1,3}=\beta_{2,2}=\beta_{2,3}=\beta_{3,1}=\mathrm{id}, \quad \beta_{1,2}=\beta_{2,1}=\beta_{3,2}=\beta_{3,3}=(a b) .
$$

is a constant cocycle of $X$. To compute the extension $S \times_{\beta} X$, let

$$
x_{j}= \begin{cases}(a, j) & \text { if } j \in\{1,2,3\}, \\ (b, j-3) & \text { if } j \in\{4,5,6\} .\end{cases}
$$

Then $S \times_{\beta} X$ is the cycle set over $\left\{x_{1}, \ldots, x_{6}\right\}$ given by

$$
\begin{gathered}
\psi_{x_{1}}=\psi_{x_{4}}=\left(x_{2} x_{5}\right), \\
\psi_{x_{2}}=\psi_{x_{5}}=\left(x_{1} x_{4}\right), \\
\psi_{x_{3}}=\psi_{x_{6}}=\left(x_{1} x_{2} x_{4} x_{5}\right)\left(x_{3} x_{6}\right) .
\end{gathered}
$$

Example 3.4. For a finite abelian group $A$ (written additively) we consider constant cocycles $\beta: X \times X \rightarrow \operatorname{Sym}(A)$ of the form

$$
\beta_{x, y}(b)=b+f(x, y),
$$

where $f: X \times X \rightarrow A$ is a map. By Lemma 2.1, $A \times X$ with

$$
(a, x) \cdot(y, b)=(b+f(x, y), x \cdot y), \quad a, b \in A, x, y \in X,
$$

is a cycle set if and only if

$$
f(x, z)+f(x \cdot y, x \cdot z)=f(y, z)+f(y \cdot x, y \cdot z)
$$

for all $x, y, z \in X$. Such a map $f$ will be called a cocycle of $X$ and it will be used to construct a cycle set structure over $A \times X$. 
Remark 3.5. The cocycles of Example 3.4 are related to the homology theory for set-theoretic solutions of the Yang-Baxter equation of Carter, Elhamdadi and Saito. This homology is useful to construct invariants of virtual knots, see for example [2, §2] and [6].

Cocycles like those of Example 3.4 are often easy to compute. To compute all the cocycles of a cycle set $X$ with values in, say, the finite field with $q$ elements $A=\mathbb{F}_{q}$, one needs to solve a linear system (over $\mathbb{F}_{q}$ ) with $|X|^{3}$ equations and $|X|^{2}$ unknowns.

Example 3.6. Let us write $\mathbb{F}_{2}=\{0,1\}$. Let $X=\{1,2,3\}$ be the cycle set given by the permutations

$$
\varphi_{1}=\varphi_{2}=\mathrm{id}, \quad \varphi_{3}=(12),
$$

and $f: X \times X \rightarrow \mathbb{F}_{2}$ be the cocycle given by

$$
f(x, y)= \begin{cases}1 & \text { if }(x, y)=(3,2) \\ 0 & \text { otherwise }\end{cases}
$$

For $j \in\{1, \ldots, 6\}$ let

$$
x_{j}= \begin{cases}(0, j) & \text { if } j \in\{1,2,3\}, \\ (1, j-3) & \text { if } j \in\{4,5,6\} .\end{cases}
$$

The extension of $X$ by $f$ is the cycle set structure over $\left\{x_{1}, \ldots, x_{6}\right\}$ given by

$$
\psi_{x_{1}}=\psi_{x_{2}}=\psi_{x_{4}}=\psi_{x_{5}}=\mathrm{id}, \quad \psi_{x_{3}}=\psi_{x_{6}}=\left(x_{1} x_{2} x_{4} x_{5}\right) .
$$

Example 3.7. Let $X=\{1,2,3,4,5\}$ be the cycle set defined by

$$
\varphi_{1}=\varphi_{2}=(45), \quad \varphi_{3}=(1425), \quad \varphi_{4}=\varphi_{5}=(12),
$$

and let $f: X \times X \rightarrow \mathbb{F}_{2}$ be the cocycle given by the matrix

$$
F=\left(\begin{array}{lllll}
0 & 0 & 0 & 0 & 0 \\
0 & 0 & 0 & 0 & 0 \\
0 & 1 & 0 & 0 & 1 \\
1 & 1 & 0 & 0 & 0 \\
1 & 1 & 0 & 0 & 0
\end{array}\right)
$$

(This means that $f(x, y)$ is the $(x, y)$-entry of the matrix $F$.) Let us compute the extension of $X$ by $f$. For $j \in\{1, \ldots, 10\}$ let

$$
x_{j}= \begin{cases}(0, j) & \text { if } 1 \leq j \leq 5, \\ (1, j-5) & \text { if } 6 \leq j \leq 10 .\end{cases}
$$

The extension of $X$ by $f$ is the cycle set over $\left\{x_{1}, x_{2}, \ldots, x_{10}\right\}$ given by

$$
\begin{gathered}
\psi_{x_{1}}=\psi_{x_{2}}=\psi_{x_{6}}=\psi_{x_{7}}=\left(x_{4} x_{5}\right)\left(x_{9} x_{10}\right), \\
\psi_{x_{3}}=\psi_{x_{8}}=\left(x_{1} x_{4} x_{2} x_{10}\right)\left(x_{5} x_{6} x_{9} x_{7}\right), \\
\psi_{x_{4}}=\psi_{x_{5}}=\psi_{x_{9}}=\psi_{x_{10}}=\left(x_{1} x_{7}\right)\left(x_{2} x_{6}\right) .
\end{gathered}
$$

Example 3.8. Let us write $\mathbb{F}_{3}=\{0,1,2\}$. Let $X=\{1,2,3,4\}$ be the cycle set given by

$$
\varphi_{1}=\varphi_{2}=\varphi_{3}=\mathrm{id}, \quad \varphi_{4}=(23)
$$


and $f: X \times X \rightarrow \mathbb{F}_{3}$ be the cocycle given by

$$
f(x, y)= \begin{cases}2 & \text { if } x=y \text { or } y=4, \\ 1 & \text { if }(x, y)=(4,3), \\ 0 & \text { otherwise. }\end{cases}
$$

For $j \in\{1, \ldots, 12\}$ let

$$
x_{j}= \begin{cases}(0, j) & \text { if } 1 \leq j \leq 4 \\ (1, j-4) & \text { if } 5 \leq j \leq 8, \\ (2, j-8) & \text { if } 9 \leq j \leq 12 .\end{cases}
$$

The extension of $X$ by $f$ is the cycle set over $\left\{x_{1}, x_{2}, \ldots, x_{12}\right\}$ given by

$$
\begin{gathered}
\psi_{x_{1}}=\psi_{x_{5}}=\psi_{x_{9}}=\left(x_{1} x_{9} x_{5}\right)\left(x_{4} x_{12} x_{8}\right), \\
\psi_{x_{2}}=\psi_{x_{6}}=\psi_{x_{10}}=\left(x_{2} x_{10} x_{6}\right)\left(x_{4} x_{12} x_{8}\right), \\
\psi_{x_{3}}=\psi_{x_{7}}=\psi_{x_{11}}=\left(x_{3} x_{11} x_{7}\right)\left(x_{4} x_{12} x_{8}\right), \\
\psi_{x_{4}}=\psi_{x_{8}}=\psi_{x_{12}}=\left(x_{2} x_{3} x_{6} x_{7} x_{10} x_{11}\right)\left(x_{4} x_{12} x_{8}\right) .
\end{gathered}
$$

3.2. Counterexamples to the Gateva-Ivanova conjecture. We use a dynamical extension of the square-free solution over $\{1,2\}$ to produce counterexamples to the conjecture.

Example 3.9. Let us consider the cycle set over the set $Y=\{1,2\}$ given by $\varphi_{1}=\varphi_{2}=\mathrm{id}$ and let $S=\{a, b, c, d\}$ be a set with four elements. The map $\alpha: Y \times Y \times S \rightarrow \operatorname{Sym}(S)$ given by

$$
\begin{gathered}
\alpha_{1,1}(a,-)=\alpha_{1,1}(b,-)=\alpha_{2,2}(a,-)=\alpha_{2,2}(b,-)=(c d), \\
\alpha_{1,1}(c,-)=\alpha_{1,1}(d,-)=\alpha_{2,2}(c,-)=\alpha_{2,2}(d,-)=(a b), \\
\alpha_{1,2}(a,-)=\alpha_{1,2}(c,-)=\alpha_{2,1}(a,-)=\alpha_{2,1}(c,-)=\mathrm{id}, \\
\alpha_{1,2}(b,-)=\alpha_{1,2}(d,-)=\alpha_{2,1}(b,-)=\alpha_{2,1}(d,-)=(a c)(b d),
\end{gathered}
$$

is a dynamical cocycle of $Y$. For $j \in\{1, \ldots, 8\}$ let

$$
x_{j}= \begin{cases}(a, j) & \text { if } 1 \leq j \leq 2, \\ (b, j-2) & \text { if } 3 \leq j \leq 4, \\ (c, j-4) & \text { if } 5 \leq j \leq 6, \\ (d, j-6) & \text { if } 7 \leq j \leq 8\end{cases}
$$

Then $X=S \times_{\alpha} Y$ is the square-free cycle set over $\left\{x_{1}, \ldots, x_{8}\right\}$ given by

$$
\begin{aligned}
& \psi_{x_{1}}=\left(x_{5} x_{7}\right), \\
& \psi_{x_{2}}=\left(x_{6} x_{8}\right), \\
\psi_{x_{3}}= & \left(x_{2} x_{6}\right)\left(x_{4} x_{8}\right)\left(x_{5} x_{7}\right), \\
\psi_{x_{4}}= & \left(x_{1} x_{5}\right)\left(x_{3} x_{7}\right)\left(x_{6} x_{8}\right), \\
& \psi_{x_{5}}=\left(x_{1} x_{3}\right), \\
& \psi_{x_{6}}=\left(x_{2} x_{4}\right), \\
\psi_{x_{7}}= & \left(x_{1} x_{3}\right)\left(x_{2} x_{6}\right)\left(x_{4} x_{8}\right), \\
\psi_{x_{8}}= & \left(x_{1} x_{5}\right)\left(x_{2} x_{4}\right)\left(x_{3} x_{7}\right) .
\end{aligned}
$$


Using the cycle set $X$ we construct a counterexample to the Gateva-Ivanova conjecture: Let

$$
r: X \times X \rightarrow X \times X, \quad r\left(x_{i}, x_{j}\right)=\left(\psi_{\psi_{x_{j}}^{-1}\left(x_{i}\right)}\left(x_{j}\right), \psi_{x_{j}}^{-1}\left(x_{i}\right)\right), \quad x_{i}, x_{j} \in X .
$$

Since $\psi_{x}^{-1}=\psi_{x}$ for all $x \in X$ and $\psi_{\psi_{y}(x)}(y)=\psi_{x}(y)$ for all $x, y \in X$, it follows that

$$
r\left(x_{i}, x_{j}\right)=\left(\psi_{x_{i}}\left(x_{j}\right), \psi_{x_{j}}\left(x_{i}\right)\right) .
$$

The solution $(X, r)$ satisfies $\operatorname{Ret}(X, r)=(X, r)$ and hence $(X, r)$ is a squarefree solution which is not a multipermutation solution.

Remark 3.10. In [4, Cor. 2.9] Cedó, Jespers and Okniński proved that every square-free solution with an abelian Yang-Baxter permutation group is a multipermutation solution. The Yang-Baxter permutation group of the solution $(X, r)$ of Example 3.9 is isomorphic to

$$
\langle(57),(68),(26)(48)(57),(15)(37)(68),
$$

$$
(13),(24),(13)(26)(48),(15)(24)(37)\rangle \simeq \mathbb{D}_{4} \times \mathbb{D}_{4},
$$

where $\mathbb{D}_{4}$ denotes the dihedral group of 8 elements.

We conclude the paper with more counterexamples to the Gateva-Ivanova conjecture. These counterexamples are constructed as extensions of the solution $(X, r)$ of Example 3.9. The key ingredient is a result of Cedó, Jespers and Okniński about liftings of multipermutation solutions, see [5, Lemma 4]. This lemma states that if $Y \rightarrow Z$ is a surjective homomorphism of solutions and $Y$ is a multipermutation solution then so is $Z$.

We say that a cycle set $X$ is a multipermutation cycle set if its corresponding solution $\left(X, r_{X}\right)$ is a multipermutation solution.

Lemma 3.11. Let $X$ be a finite square-free cycle set, let $S$ be a nonempty set and let $\alpha \in Z^{2}(X, S)$ with $\alpha_{x, x}(s, s)=s$ for all $x \in X$ and $s \in S$. Assume that $X$ is not a multipermutation cycle set. Then $S \times_{\alpha} X$ is square-free and it is not a multipermutation cycle set.

Proof. The dynamical extension $S \times_{\alpha} X$ of the cycle set $X$ is a square-free cycle set by Lemma 2.1 and Remark 2.6. It induces a surjective homomorphism of solutions

$$
(S \times X) \times(S \times X) \rightarrow X \times X, \quad((s, x),(t, y)) \mapsto(x, y) .
$$

Thus the claim follows from [5, Lemma 4].

Acknowledgement. The author wishes to thank Ferran Cedó for comments on the paper, Travis Schedler for the list of nondegenerate involutive set-theoretic solutions of size $\leq 8$ and the ICTP for the support and for the working atmosphere.

\section{REFERENCES}

[1] N. Andruskiewitsch and M. Graña, From racks to pointed Hopf algebras, Adv. Math. 178 (2003), no. 2, 177-243. MR1994219 (2004i:16046)

[2] J. S. Carter, M. Elhamdadi, and M. Saito, Homology theory for the set-theoretic YangBaxter equation and knot invariants from generalizations of quandles, Fund. Math. 184 (2004), 31-54. MR2128041 (2005k:57009) 
[3] F. Cedó, E. Jespers, and Á. del Río, Involutive Yang-Baxter groups, Trans. Amer. Math. Soc. 362 (2010), no. 5, 2541-2558. MR2584610 (2011i:16058)

[4] F. Cedó, E. Jespers, and J. Okniński, Retractability of set theoretic solutions of the Yang-Baxter equation, Adv. Math. 224 (2010), no. 6, 2472-2484. MR2652212 (2011f:16093)

[5] _ Braces and the Yang-Baxter equation, Comm. Math. Phys. 327 (2014), no. 1, 101-116. MR3177933

[6] J. Ceniceros and S. Nelson, Virtual Yang-Baxter cocycle invariants, Trans. Amer. Math. Soc. 361 (2009), no. 10, 5263-5283. MR2515811 (2010k:57021)

[7] F. Chouraqui and E. Godelle, Finite quotients of groups of I-type, Adv. Math. 258 (2014), 46-68. MR3190423

[8] P. Dehornoy, Coxeter-like groups for set-theoretic solutions of the Yang-Baxter equation, C. R. Math. Acad. Sci. Paris 351 (2013), no. 11-12, 419-424. MR3090121

[9] V. G. Drinfel'd, On some unsolved problems in quantum group theory, Quantum groups (Leningrad, 1990), 1992, pp. 1-8. MR1183474 (94a:17006)

[10] P. Etingof, T. Schedler, and A. Soloviev, Set-theoretical solutions to the quantum Yang-Baxter equation, Duke Math. J. 100 (1999), no. 2, 169-209. MR1722951 (2001c:16076)

[11] T. Gateva-Ivanova, A combinatorial approach to the set-theoretic solutions of the Yang-Baxter equation, J. Math. Phys. 45 (2004), no. 10, 3828-3858. MR2095675 (2005h:16077)

[12] _ Quadratic algebras, Yang-Baxter equation, and Artin-Schelter regularity, Adv. Math. 230 (2012), no. 4-6, 2152-2175. MR2927367

[13] T. Gateva-Ivanova and P. Cameron, Multipermutation solutions of the Yang-Baxter equation, Comm. Math. Phys. 309 (2012), no. 3, 583-621. MR2885602

[14] T. Gateva-Ivanova and S. Majid, Set-theoretic solutions of the Yang-Baxter equation, graphs and computations, J. Symbolic Comput. 42 (2007), no. 11-12, 1079-1112. MR2368074 (2009a:16078)

[15] — Matched pairs approach to set theoretic solutions of the Yang-Baxter equation, J. Algebra 319 (2008), no. 4, 1462-1529. MR2383056 (2009a:16079)

[16] _ Quantum spaces associated to multipermutation solutions of level two, Algebr. Represent. Theory 14 (2011), no. 2, 341-376. MR2776789 (2012h:16072)

[17] T. Gateva-Ivanova and M. Van den Bergh, Semigroups of I-type, J. Algebra 206 (1998), no. 1, 97-112. MR1637256 (99h:20090)

[18] W. Rump, A decomposition theorem for square-free unitary solutions of the quantum Yang-Baxter equation, Adv. Math. 193 (2005), no. 1, 40-55. MR2132760 (2005k:81132)

[19] _ Braces, radical rings, and the quantum Yang-Baxter equation, J. Algebra 307 (2007), no. 1, 153-170. MR2278047 (2007m:16065)

[20] - Semidirect products in algebraic logic and solutions of the quantum YangBaxter equation, J. Algebra Appl. 7 (2008), no. 4, 471-490. MR2442072 (2010a:81109)

Depto. de Matemática, FCen, Universidad de Buenos Aires, Pabellón I, Ciudad Universitaria (1428) Buenos Aires, Argentina

E-mail address: lvendramin@dm.uba.ar 